\title{
Nasal Tip Abscess: A Rare Infection of the Head and Neck
}

\author{
Guakil-Haber Alberto ${ }^{1}$, Kuri-García, Anuar², Espinosa-Mancilla, Angélica Eloisa ${ }^{3}$, Olvera-Suárez, María Yazmín ${ }^{4}$ \\ ${ }^{1}$ Physician, Department of Otolaryngology and Head and Neck Surgery, ${ }^{2}$ Physician, Department of Otolaryngology and \\ Head and Neck Surgery, ${ }^{3}$ Physician, Department of Otolaryngology and Head and Neck Surgery, ${ }^{4}$ Professor, Department of \\ Otolaryngology and Head and Neck Surgery, Centro Médico ISSEMyM Toluca, Universidad Autónoma del Estado de México.
}

Corresponding author: Guakil-Haber Alberto, Physician, Department of Otolaryngology and Head and Neck Surgery,Centro Médico ISSEMyM Toluca, Universidad Autónoma del Estado de México.

DOI: http://dx.doi.org/10.21276/ijcmsr.2019.4.4.35

How to cite this article: Guakil-Haber, Alberto, Kuri-García, Anuar, Espinosa-Mancilla, Angélica Eloisa, OlveraSuárez, María Yazmín. Nasal tip abscess: a rare infection of the head and neck. International Journal of Contemporary Medicine Surgery and Radiology. 2019;4(4):D150-D151.

\section{A B S T R A C T}

Introduction: The nasal tip abscess is a rare pathology that affects the soft tissue of the nasal tip, with risk of damaging the cartilaginous framework with the resulting alteration of the anatomy and function of the nose, if not treated properly. Epidemiologic features have not been extensively characterized in the international literature because of its low incidence. Case report: We present a case report of a nasal abscess secondary to furunculosis in a patient with long evolution and uncontrolled type 2 diabetes presented as nasal swelling with erythema that did not respond to oral antibiotics. Treatment consisting of topic and systemic antibiotic therapy, as well as surgical drainage, which requires ample knowledge of nasal tip anatomy, was used.

Conclusion: In our case, resolution was achieved without sequels or scarring.

Keywords: Abscess, Drainage, Infection, Nasal Tip, Nose.

\section{INTRODUCTION}

The nose can be divided in three thirds: superior, middle and inferior. The superior third consists fundamentally on the bony pyramid, formed by the nasal bones and frontal processes of the maxillary bone. The middle third is formed by the cartilaginous vault, made up by the cartilaginous septum and the upper lateral cartilages or triangular cartilages. Finally, the lower third is the formed by the nasal tip. ${ }^{1}$

The nasal tip can be defined as the lower third or inferior fifth of the nose. Also, the tip can be divided in lobule, soft tissue areas, alar cartilages and columella. These structures conform a cartilaginous pyramid formed by the alar cartilages, subcutaneous tissue, in which blood vessels and nerves can be found, and it's attached to the superficial musculoaponeurotic system, formed the fascia of the nasal musculature. ${ }^{2}$

The skin of the nose has different thickness depending on location the upper third is the thinnest end the tip the thickest, containing more subcutaneous tissue a sebaceous glands. ${ }^{1}$ This can have a crucial role in the mechanism in which infections evolve to an abscess.

\section{Nasal tip abscess}

Nasal tip abscess is defined as an infection confined to the lower third of the nose, which can be derived from different causes. The most common is after rhinoplasty, deriving from the sutures ${ }^{3}$, after nasal filler injections ${ }^{4}$, and rarely secondary to furunculosis. ${ }^{5}$

We present a case of nasal tip abscess secondary to furunculosis, it's clinical presentation and management.

\section{CASE REPORT}

A 53-year-old male patient with history of smoking, alcoholism and 15 years of poorly controlled type 2 diabetes, he presented for evaluation of a 1 week history of swelling, pain, hyperthermia and erythema of the nasal tip, he was treated with clindamycin and ceftriaxone by his family physician, but despite treatment his symptoms deteriorated and referral to our otolaryngology and head and neck surgery was made. Upon physical examination an important swelling of the nasal tip was found accompanied by hyperthermia, erythema (Rudolph sign) (Figure 1a), intense pain and fluctuanting consistency, asymmetric vestibules and deviated aedematous columnella, a deviated septum with a right basal crest and spur were also found. Aedema was seen in Cottle I area as seen in Figure 2.

Laboratory studies on admission showed white blood cell count of $13 \times 10^{3} / \mu \mathrm{L}, 89 \%$ neutrophils, hemoglobin of $15.3 \mathrm{~g} /$ $\mathrm{dL}$, hematocrit $46 \%$, platelet count of $284 \times 10^{3} / \mu \mathrm{L}$, fasting glucose of $195 \mathrm{mg} / \mathrm{dl}$, anti-HIV antibodies were negative. Surgical drainage was performed using a left marginal incision approach (Figure 3), soft tissue dissection was made with a blunt tip scissor over the alar cartilages, columella, nasal tip and nasal dorsum, taking care not to injure the cartilages, obtaining $5 \mathrm{cc}$ of purulent material, which was sent to the laboratory for culture. Surgical debridement followed by antiseptic hyperchlorinated water irrigation was done, leaving the wound open for irrigation and secondary closure. Placement of a splint to manage aedema, oral antibiotics 


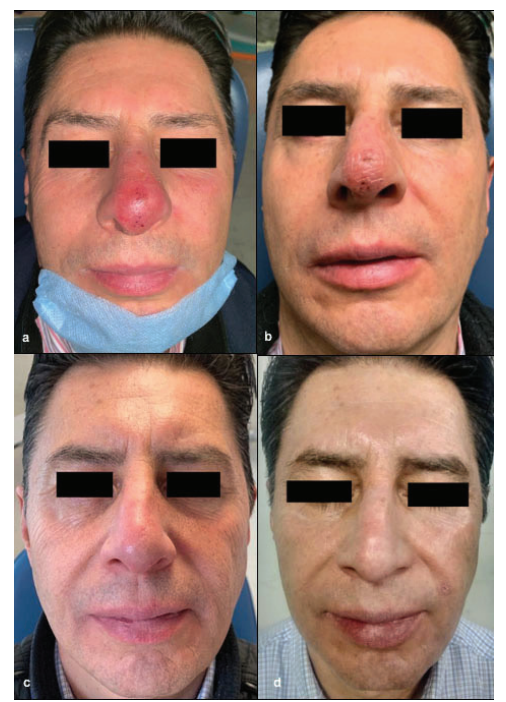

Figure-1: Clinical evolution.
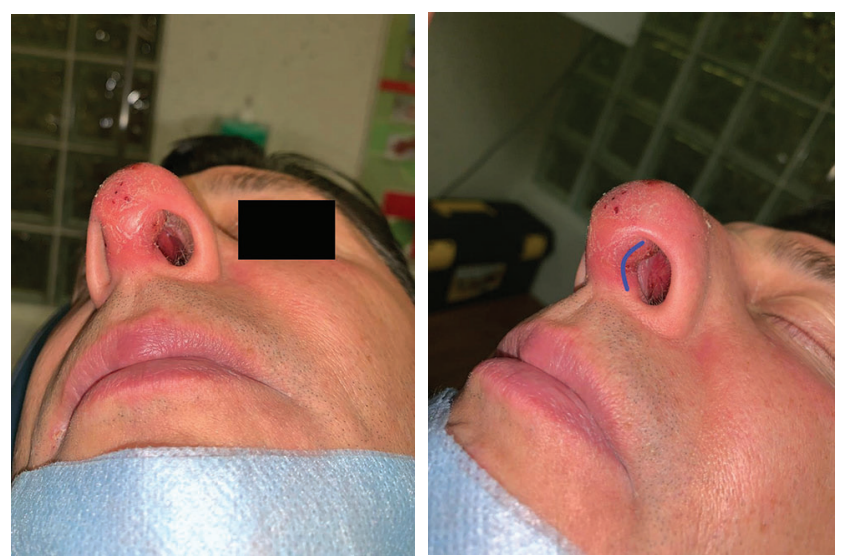

Figure-2: Inferior nasal tip view; Figure-3: Schematic illustration of left marginal incision approach

consisting of clyndamicin and dicloxacyline were given. After 3 days of daily debridement and lavage of the wound, the patient was discharged from the hospital. Culture showed Staphylococcus aureus growth sensible to clyndamicin, oral antibiotics were continued until a 10-day treatment was completed. Patient was asymptomatic, with no clinical or laboratorial evidence of recurrence of the infection, 6 months follow up showed no sign of nasal deformity, cartilage or skin damage or abnormal scarring.

\section{DISCUSSION}

Nasal tip abscesses are a rare pathology of the head and neck, and therefore epidemiological data and clinical features have not been extensively characterized. ${ }^{3,6}$ In this case we present a nasal tip abscess secondary to furunculosis, which we found a few reports.

Furunculosis is associated with the colonization of the sebaceous glands of the nasal tip, by bacteria like Propynebacterium acnes or Staphylococcus aureus present in the skin of the nose,,$^{5,7}$ that can ultimately generate a nasal tip abscess.

Clinical presentation is consistent among these patients, the finding of the "Rudolph sign", that consists in swelling and erythema of the nasal tip. Other common findings are pain, exacerbated by manipulation and skin desquamation. ${ }^{9}$

Treatment consists in drainage and anti-staphylococcical antibiotic agents, some authors recommend nasal packing impregnated with antibiotic ointment. These cases generally have a good prognosis. , $^{3,9}$

\section{CONCLUSION}

Nasal tip abscess is rare pathology, and can present in several scenarios, for example, as a complication of nasal surgery, trauma, or a complicated localized infection like furunculosis, but despite the origin, management remains the same. Drainage and antibiotic therapy are the base of treatment. It's important to recognize this pathology to avoid delay in surgical drainage, or referral to an Otolaryngologist or another specialist that can perform it without damaging the cartilaginous vault or other structures of the nasal tip. The aim of this case report is to help clinicians recognize this rare pathology of the head and neck region and improve the outcome of these patients.

\section{REFERENCES}

1. Hsu, D.W. and J.D. Suh, Anatomy and Physiology of Nasal Obstruction. Otolaryngol Clin North Am, 2018. 51(5): 853-865.

2. Ketcham, A.S. and E.J. Dobratz, Normal and variant anatomy of the nasal tip. Facial Plast Surg, 2012. 28(2): 137-44.

3. Teymoortash, A., et al., Nasal tip abscess due to adverse skin reaction to Prolene: an unusual long term complication of rhinoplasty. J Laryngol Otol, 2013. 127(1): 76-9.

4. Lee, K.H., Infection in the Nasal Tip Caused by Acellular Dermal Matrix. Plast Reconstr Surg Glob Open, 2015.3(12): p. e581.

5. Bakshi, S.S., Image Diagnosis: Nasal Furunculosis-A Dangerous Nose Infection. Perm J, 2017. 22.

6. Cheng, J. and L.P. Smith, Intranasal Drainage for Pediatric Nasal Abscesses. Otolaryngol Head Neck Surg, 2014;151(1): 179-81.

7. Farabi Lee Jaafar, A., Nose abscess after thread rhinoplasty: a case report. 2018;2(4); 46-51.

8. Dahle, K.W. and R.D. Sontheimer, The Rudolph sign of nasal vestibular furunculosis: questions raised by this common but under-recognized nasal mucocutaneous disorder. Dermatol Online J, 2012. 18(3): p. 6.

9. Morales-Angulo C, et al., Absceso profundo de punta nasal.Deep abscess of nasal tip. Rev Soc Otorrinolaringol Castilla Leon Cantab La Rioja, 2013. 4(12): p. 106-110.

\section{Source of Support: Nil; Conflict of Interest: None}

Submitted: 22-08-2019; Accepted: 18-10-2019; Published online: 23-12-2019 